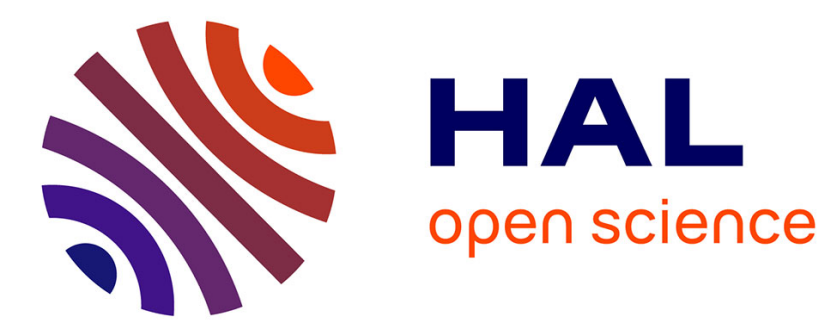

\title{
Monitoring player attention: A non-invasive measurement method applied to serious games
}

Zahen Malla Osman, Jérôme Dupire, Stéphanie Mader, Pierre Cubaud, Stéphane Natkin

\section{- To cite this version:}

Zahen Malla Osman, Jérôme Dupire, Stéphanie Mader, Pierre Cubaud, Stéphane Natkin. Monitoring player attention: A non-invasive measurement method applied to serious games. Entertainment Computing, 2016, 14, pp.33-43. 10.1016/j.entcom.2015.08.003 . hal-02456042

\section{HAL Id: hal-02456042 https://hal-cnam.archives-ouvertes.fr/hal-02456042}

Submitted on 27 Jan 2020

HAL is a multi-disciplinary open access archive for the deposit and dissemination of scientific research documents, whether they are published or not. The documents may come from teaching and research institutions in France or abroad, or from public or private research centers.
L'archive ouverte pluridisciplinaire HAL, est destinée au dépôt et à la diffusion de documents scientifiques de niveau recherche, publiés ou non, émanant des établissements d'enseignement et de recherche français ou étrangers, des laboratoires publics ou privés. 


\title{
Monitoring the Player's Attention \\ A Non Invasive Measurement Method Applied to the Serious Games
}

\author{
Zahen Malla Osman, Jérôme Dupire, Stéphanie Mader, Pierre Cubaud, \\ Stéphane Natkin \\ Centre d'Etudes et de Recherches en Informatique du CNAM \\ Conservatoire National des Arts et Métiers \\ 292 Rue Saint Martin, 75141 Paris, France
}

\begin{abstract}
In this paper, we report on an experiment to monitor the user attention in a serious game called Le Village aux Oiseaux (The Birds' Village). The main motivation of this study stems from the importance of dynamically adapting scenario and challenges to the skills of each player. The solution we developed is a real-time, non-invasive visual attention monitoring method, using an evaluation of the reaction time in an open and continuous space. Our results provide information that can be useful to manage automatically each game session. It can be of benefit to game designers as well, allowing them to use this information to improve gameplay, manage the difficulty of game environments, and optimize positioning of visual resources on screen.
\end{abstract}

Keywords: Serious games, attention, pointing task, reaction time, Alzheimer.

\section{Introduction}

Le Village aux Oiseaux (The Birds' Village) is a therapeutic game for seniors suffering from Alzheimer's disease. The game seeks to train the attention

\footnotetext{
Email addresses: zahen.malla_osman@cnam.fr (Zahen Malla Osman), jerome.dupire@cnam.fr (Jérôme Dupire), stephanie.mader@cnam.fr (Stéphanie Mader), pierre-henri.cubaud@cnam.fr (Pierre Cubaud), stephane.natkin@cnam.fr (Stéphane Natkin)
}

Preprint submitted to Entertainment Computing 
network to slow down the cognitive losses due to Alzheimer's. According to [1], attention is a function of the ability to select an object or stimulus from among many others and then to decide whether it should be ignored or whether action must be taken. For example, a vehicle horn or a door slamming can attract attention (detection) but does not necessarily require an action (interpretation). Le Village aux Oiseaux relies on the following principles: highly interactive, fun and auto adaptative.

Interactivity is the foundation of the therapy: by stimulating a lot the attention network, we expect to maintain much longer up and running the users' cognitive abilities.

Fun will make people to play the game and play again in the future. The more the user plays, the more she/he cares.

Adaptivity is necessary for the game system to propose relevant contents to the users, depending on their remaining capabilities, mood, health, motivation, etc.

To provide such efficient training, the system have to ensure that the player is focused on the game. A major challenge is to provide to the system a tool to monitor the player's attention level. Then, whenever the player loss his concentration, the game would be able to help him to (re)focus by triggering special events or to stop the session if such special events remain inefficient.

To know when the player has an attention gap, we need an approximation of their attentional capabilities to compare with. Such data is also necessary for adapting game difficulty to the player abilities, which is important to provide an adapted training, but also to foster the player's motivation. Indeed, an adapted difficulty is one of the key item to reach the flow state.

Many measures of attention rely on physiological and behavioral manifestations, such as electrodermal activity, heart rate, respiratory rate, or eye movements. Yet, Le Village aux Oiseaux will be played by elderly people at home without supervision. Consequently, we needed a method to measure attention that (1) is not invasive, (2) requires no IT skills, (3) costs as little as possible, (4) is able to detect in real time any change in the attention level of the user. 
In a video game, the user frequently moves the mouse cursor toward an object, whether it be to select an option or to kill an enemy. Our main hypothesis is that we can measure the reaction time (RT) of a user by making a geometrical and temporal analysis of the movements made by users in real time. RT is easily computed as the difference between the time corresponding to the beginning of the user's voluntary movement and the time when the target appeared. Then, we can evaluate the current user's attention level by comparing the current RT with the mean RT of the user.

The main challenge of our method lies on the fact that the system does not have any clue to determine the beginning of a movement which would be triggered by the appearance of the target (i.e. the user moves actually the cursor in order to select the target). Since the user is free to move wherever he/she wants, regardless to the presence of target on screen, the system can not determine which movement are motivated by selecting the target and which ones are not.

Our first implementation of this method consists of three experiments. First, we conducted two experiments with simple pointing tasks in a non-game environment. Then, a third experiment in a simplified version of Le Village aux Oiseaux.

This paper is organized as follows: Section 2 describes the general design of the serious game Le Village aux Oiseaux; Section 3 presents related work; Section 4 describes our experiments; Section 5 summarizes our paper and provides an outlook for future work.

\section{Game Design}

Le Village aux Oiseaux (Fig. 1) is a cognitive rehabilitation game for patients suffering from the Alzheimer's disease. Therapeutic games, to which cognitive rehabilitation game belongs, are a category of serious games that aim to improve the health condition of their players [2]. Indeed, by applying the definition of therapy to games [3], we can define therapeutic games as follow : a game whose 
intended and expected effect is to heal, to alleviate, or to improve a particular health condition.

According to Guardiola et al., a serious game is a rule-based formal system with variable and quantifiable outcomes, where different outcomes are assigned different values; the player exerts effort in order to influence the outcome, and the player feels attached to the outcome. A serious game, however, combines this with a defined, real-life objective [4]. This definition is based upon the classic game definition given by Juul [5].

It means that a serious game are above all games, but games that have been designed to attain a real-life objective. In Le Village aux Oiseaux, this objective is to provide a cognitive training to patients suffering from the Alzheimer disease. The therapeutic hypothesis on which the project was founded is that stimulating the attentional network of the patient may slow down the effects of the cognitive decline due to the disease's progression. As a result, the patient would stay at a higher level of cognitive functioning by playing Le Village aux Oiseaux.

Video games have already been used to attain such objective. Using video games leads to the improvement of cognitive functioning of elderly people [6]. Playing video games can improve the performance of the players [7] and especially the performance of elderly people [8] [9] [10].

Using games in therapy is seen as a promising solution because they can motivate the player, motivation being a major challenge in a therapy context [1] 12. Indeed, therapies are often repetitive and boring, even provoking painful side-effects. As a result, patients tend to be less regular in their therapeutic protocol, and even sometimes drop out.

Indeed, video game is a type of application that attracts a large number of users across the world. According to Pew Internet and American Life Survey 2008 [13] [14, more than half of all American adults play video games. Facing challenges and being immersed in an interesting game world are two of the main reasons why players play [15] [16].

In this article, we focus on the challenges and their difficulty, but we also designed a game world, with a story and other characters for the player to meet. 
Here is a resume to give some context.

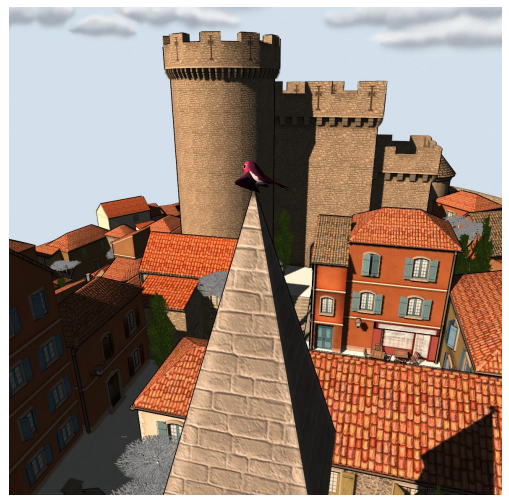

(a) Top-down view of the castle

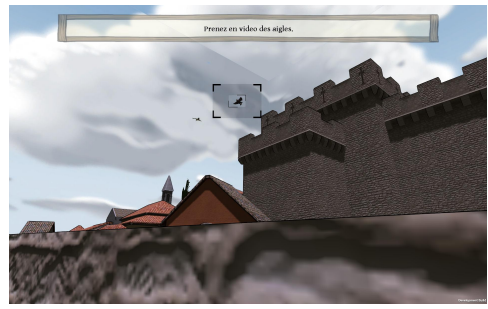

(c) In-game screen-shot

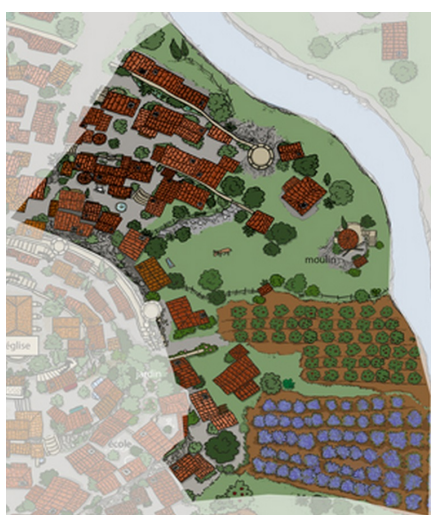

(b) General map

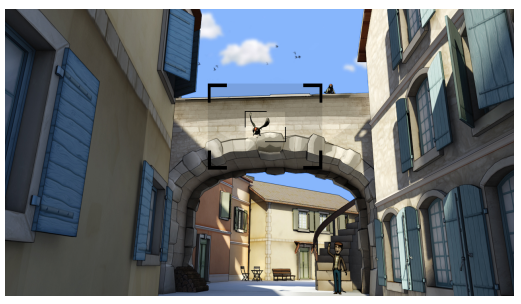

(d) In-game screen-shot

Figure 1: Screen-shots of Le Village aux Oiseaux: top-down view of the castle (a), general map (b), in-game pictures (c, d).

In Le Village aux Oiseaux, the player takes the role of a photograph arriving in a little Provencal town about to be destroyed by a real estate project. To help the villagers, the player will take pictures of birds to prepare a report proving that numerous birds live around the village. Thus giving the town the status of nature reserve and leading to the interruption of the real estate project.

First, we briefly explain how we choose and adapted a gameplay for $L e$ Village aux Oiseaux, then we explain our approach to the difficulty and why it is necessary to have an estimate of the attentional capabilities of the player. 


\subsection{Designing the gameplay}

A gameplay is composed of (1) challenges that the player faces, (2) the actions the player can do to address the challenge [17. Challenges can be of different types regarding the kind of actions that the player has to do: sensitive / perceptive, cognitive (logical), motor [18]. In therapeutic games, the gameplay is what provide the therapeutic effect, and thus is the most important aspect to design.

Moreover, in addition to provide the therapeutic effect, the gameplay should motivate the players and be accessible to them. Here, our players are seniors with different degree of cognitive loss, meaning the accessibility should be though from the beginning.

As a consequence, we first selected a gameplay that can provide the therapeutic effect, then we adapted it to our players, and afterwards we designed additional part of the game to foster the player motivation.

The first design decisions were based on a study of Green and Bavelier, who found out that players of first person shooter games were better at different visual attentional tasks because the game trained their attention network [19]. In a later study, Dye, Green, and Bavelier discovered that players of action games, to which first person shooter games belong, had improved their attention network, making them faster and more efficient than non-players [20].

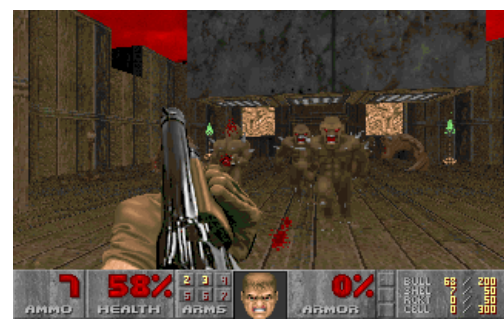

Figure 2: Doom: a first person shooter game

Therefore, the gameplay chosen for Le Village aux Oiseaux is the gameplay of first person shooter game (Fig. 2). This implies (i) that the main mechanics of the game is to aim at targets on the screen and validating it by pressing on a 
button at the right time (i.e. while the crosshair is aligned with the target), and (ii) that the game world is a 3 -dimensionals (3D) environment seen through the eyes of the avatar (i.e. first person perspective). However, first person shooter games are generally conceived for young male adults or adolescents, which is why this gameplay must be adapted to be accessible to and engaging for seniors [21].

According to our insights, experience and an analysis of our purpose, we defined that we had to simplify the gameplay and the game controls. Indeed, moving and orienting the avatar in a 3D environment is a difficult task that requires some training. It requires strong abilities in spatial orientation and representation, as well as learning complex control schemes. Meaning that the player can lose himself in the game environment, but also that he has to manipulate at the same time the two analog sticks of a game controller, one to move his avatar and one to orient his view.

Learning this task is tedious and seniors are seldom experimented with the task. To minimize the probability of our player rejecting the game because of this tedious learning, we decided to automatize this part of the gameplay.

Thus, the avatar follows a predefined rail, making the player's experience similar to the one of a roller coaster. The rail defines the path of the avatar in the 3D game world and where it looks. It also defines at which speed the avatar moves, when it stops and for how long. Therefore, Le Village aux Oiseaux belongs to a sub-category of first person shooter games called rail shooter, like the game Ghost Squad (Fig. 3).

By having a gameplay focused on the aim mechanic, the game is accessible to seniors while keeping a 3D environment. Indeed, aiming is mainly a $2 \mathrm{D}$ task, the player's challenge is to position his cursor upon the target's $\mathrm{x}$ and $\mathrm{y}$ positions (relative to the screen).

Moreover, rail shooters are primarily played with game controllers like Light Gun, Sony PSMove, or Nintendo WiiMote, simplifying the control scheme and make the aim more efficient and intuitive, so quickly enjoyable for the player $[22$. 

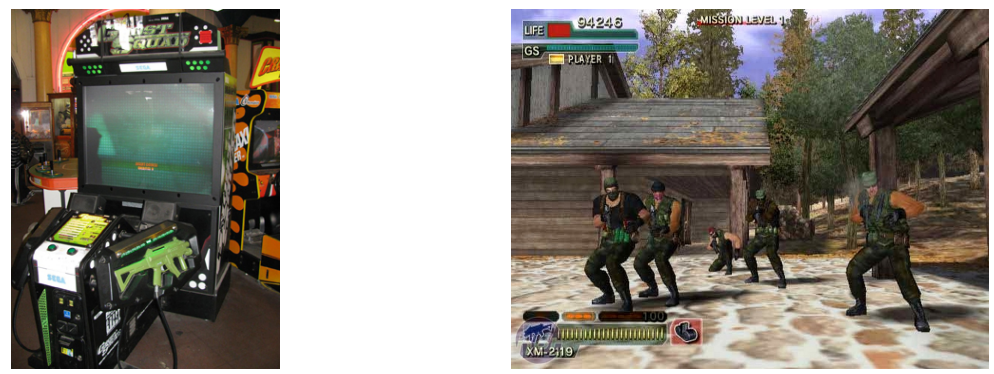

Figure 3: A famous rail shooter: Ghost Squad

Finally, having a camera on rail gives us a stronger control on the player experience. Having a complete control over where the player's character is and looks in the game world gives the possibility of regulating the rhythm of the game to help the player keep his attention focused on it. It is then possible to prepare more or less difficult game situations, and to include a more supervised storytelling within the game level. Finally, game parameters such as the character's speed or the duration of game situations can be changed by an adaptive difficulty system taking into account the player's profile (i.e. how well the player is performing).

As our design decision were based on four hypotheses, we verified them by organizing a playtest. We chosed existing games and observed seniors playing them to assess if:

- Seniors like games in which they have to aim a target and validate it.

- Seniors are able to use a game controller similar to a Nintendo Wiimote, because it is similar to a TV controller (buttons, shaping, action of pointing in the direction of the screen).

- Seniors adapt themselves to the use of such controller to point to targets on the screen.

- Seniors have difficulties to move and orient a character in a 3D environment. 
The playtest are explained in details in [2]. Our observation confirmed that the gameplay we selected and the adaptations we made to it should result in an accessible and enjoyable game to seniors.

\subsection{Designing the difficulty}

To be effective, a cognitive training or rehabilitation program has to be adapted to the patient's abilities. A balanced level of difficulty (i.e. neither too easy nor too difficult) is also an important factor in motivation, as well as being necessary to attain a state of "flow" 223. The "flow" state is a state of momentum within which someone is completely concentrated on the task at hand.

Even if challenges have a specific level of difficulty, a moderate challenge can be too easy for an expert and too hard for a beginner. As a consequence, the actual difficulty level of a challenge is dependent of the capabilities of the player [18. Thus, we need to assess the player capabilities to be able to set an effective difficulty level for him. In Le Village aux Oiseaux, it means being able to assess his attention capabilities.

Le Village aux Oiseaux is designed to be played by elderly people in their homes without supervision. Thus, our method to evaluate attention should be straightforward for the patient, meaning a method requiring no specific devices or sensors. If the last sections explained how we designed and validated our system to measure the player attention, this section explain how we use the player profile in Le Village aux Oiseaux to adapt the difficulty level of the challenges.

The main challenge of Le Village aux Oiseaux is to photography birds, but the player get instructions about which kind of birds he has to photograph and at which moment (i.e. while the bird is doing something in particular). An example of instruction would be: Take 10 pictures of swallow while they are flying.

The gameplay is composed of two kind of actions. Explicit actions that is actions whose have a visible manifestation such as pressing a button to take the 
picture, and implicit actions whose are never known by the game system such as detecting a bird or thinking.

To complete a photography challenge, the player has to (1) observe the screen to detect the presence of birds, (2) follow by sight what a detected bird is doing, (3) take a picture when the right bird is doing the right behavior.

Those 3 steps can be decomposed in more detailed actions. For instance, to take the picture, the player has to aligned the cursor with the bird, and then to press a button at the right moment to take the picture.

By listing every actions of these 3 steps, we have been able to determine that the most important actions for the therapeutic effects were connected to the observation and the following of birds, and that taking the picture was mainly a motor challenge. Thus, we have two kinds of difficulty level, one for the therapy, and one for the motor challenge.

This separation is also convenient because our players, being seniors, may have other health condition that may impact their motor skills such as arthritis. Being able to adapt the difficulty with such details is necessary to provide an accessible and enjoyable game for everyone.

Indeed, it is not because a senior is very slow to take a picture that he cannot detect the birds very quickly when they appear on the screen. Conversely, a senior being slow to detect birds can be very quick when it comes to taking the pictures.

As we know every implicit and explicit actions the player has to do to complete the challenge, we can determine for each of them what are the parameters that we can use to modify the difficulty level. For instance, detecting a bird is more or less difficult depending on its size on screen, its contrast to the background, and its distance to the attention focus of the player. Identifying a bird is more or less difficult regarding the number of different birds existing in the game and their difference (size, color, shape).

The adaptation of the motor challenge difficulty can easily been done by changing the size of the cursor (i.e. the bigger the easier), but other means can be used such as slowing the birds. The way by which we can infer the motor 
capabilities of the player is still under design and out of the scope of this paper.

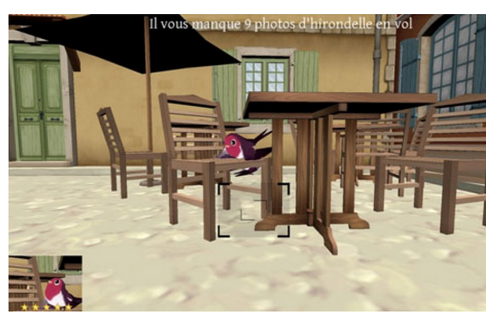

(a) Average level

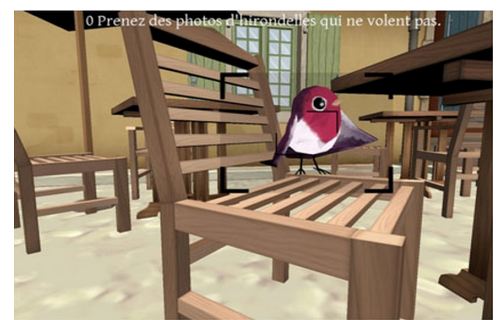

(b) Easy level

Figure 4: Two levels of difficulty based on the camera zoom: average(a) and easy(b).

An attention measure system is necessary to obtain an estimate of the attentional capabilities of the player, and then to vary the difficulty parameters according to this estimation (Fig. 4). Another important aspect of such system would be to determine the moments when the player is tired or bored, in order to add events in the game to capture back his attention. Such events should be a combination of sound and visual effects such as a flock of birds passing over its head while singing.

\section{Related work}

Analyzing user behavior could allow us to monitor the cognitive status of users when they play video games.

Attention measures can be applied in serious games that are primarily designed as tools for learning: e.g. we can imagine an artificial intelligence that adapt to the game's environment based on user behavior.

Survey subjects through video games can monitor the process of attention deficit/hyperactivity disorder [24]. Early identification of cognitive decline is very useful in the elderly world to refer to the imminent functional impairment and to provide more efficacy with the treatments and/or delay disease progression [25] 26].

Many methods have been proposed for analyzing the trajectory curves of users during pointing movement. The velocity profile can characterize the tra- 
jectory made by the user; Viviani and al. 27] study gesture recognition performance by calculating the tangential velocity of the trajectory made by the user. Hourcade and al. 28] slow down the velocity of the mouse cursor when the user tries to point at a particular target on the screen. Khalid and al. 29] study the difficulties of handwriting and indicate that users who have more than one peak in their velocity profile have perturbation during the execution of hand movement. Renau and al. [30] extract several procedural features (type, orientation, structural relation ... etc.) from trajectory curves to determine and describe the execution phases of a drawing gesture. Wonner and al. [31] predict the position of a target using trajectory analysis, by extracting many dynamic features (e.g. velocity, acceleration and distance).

Testing RT is very important for detecting cognitive decline and for improving processing speed. Fitts [32] proposes a law that predicts the time required to move from an initial position to a final destination. Fitts' law depends on the distance required to point towards the target and on the size of the target. The RT is the time interval between the appearance of the stimulus and the beginning of movement toward that stimulus [33. Today, the use of RT to infer the nature of perceptual and cognitive processes is fundamental in experimental psychology.

In this paper, we report on our experiments measuring the RT during a pointing task. These experiments constitute a step towards our goal of evaluating the attention of Alzheimer's patients playing the therapeutic game Le Village aux Oiseaux.

In the current prototype of Le Village aux Oiseaux, the player takes the role of a photographer who takes pictures of birds displayed on the game screen, using the mouse as a pointing device [2]. When the user makes a movement towards the bird (target) to take a picture of it the real time analysis of this movement allows us to compute RT, which provides an indication of the attention level of the user. Then, by comparing the current RT with the mean RT of the users, it is possible to determine the loss of attention at any time during the game and thereby detect when the user begins to become tired. At such a moment, the 
game should help the user maintain concentration by rendering more visual and audio effects, such as making a flock of birds flying just over the their head. The mean RT can also be used to configure the difficulty parameters of Le Village aux Oiseaux.

\section{Method And Experiment}

The aim of the following experiment is to compute reaction time (RT) during a pointing task in a complex environment, where the user's trajectory is free within the space. Given that users use only a mouse to execute the pointing task, the moment at which the movement toward the target begins is not easy to find. We are obliged, therefore, to use data generated by the software to analyze users' trajectory and compute RTs that can provide us with an indicator of user attention.

The methods used in our experiments are based on the BAWL battery (Batterie Attention William Lennox) [34. The BAWL battery is a tool similar to TAP battery (Tests of Attentional Performance) [35. While it has been developed with neuropsychological goals in mind, it can also be useful in other domains: psychiatry, cognitive psychology, gerontology, to provide additional information following physical examination, etc. Int the way it is structured it targets more specifically the assessment of adult subjects (there are no norms for child populations). The different tests of the battery have in common the use of simple tasks, soliciting a minimal level of cognitive processing. The majority of the tasks have been constructed from a common structure (type of items, inter-stimuli intervals and presentation modalities) in order to favor reliability

in comparisons between tasks. The low level of cognitive processing linked to the simplicity of the tasks and the common structure of most of the proposed tasks improve the control of the implicated variables when analyzing a subject's performance. The battery includes a total of ten RT tasks. Most of these tasks exist in different versions, differentiated mostly by the number of items of which they are composed. The RTs are recorded in milliseconds. 
Our experiment consists of three parts: Static Pointing, which computes a reliable RT using an ideal conditions wherein users must react directly and quickly facing the visual target; Dynamic Pointing, which computes the RT within a complex environment in which the user's trajectory is free within the space; Game Pointing, where we can apply our dynamic method to compute the $\mathrm{RT}$ in the context of video games.

A total of 15 volunteers (9 male and 6 female) participated in this experiment. Their ages varied between 25 and 40 years, with a mean of 30.2. All participants are right-handed, healthy and have normal or corrected-to-normal vision. This experiment was performed on a desktop personal computer (Intel $3 \mathrm{GHz}$ Core 2 Duo, 4GB RAM) running Windows 7 Professional, with an LCD display. The participants' mouse movements were sampled at a frequency of $100 \mathrm{~Hz}$.

\subsection{Procedures}

\subsubsection{Static Pointing}

During a pointing task in a 2D graphical user interface, users can use a mouse to point at certain targets displayed on the screen. Sometimes, users will wait for the target to appear before moving their mouse towards it to achieve the task. In this case, if the user did not move his or her mouse until the target appeared and if the movement post-appearance was directly toward this target, the RT can be described by the following equation:

$$
R T=T_{\text {target }}-T_{0}
$$

Where $T_{\text {target }}$ is the moment of the target's appearance and $T_{0}$ is the moment of beginning the movement toward this target.

In this task, users must move the mouse cursor towards a white square located at the center of the pointing application interface $(1000 \times 1000$ pixels $)$, and preserve the mouse cursor without any movement inside this white square during a random period of time ranging from 1 to 3 seconds. After the target's 
appearance on the interface, the white square disappears automatically and the user must make a direct movement towards the target to point at it.

The waiting period is built in order to display the target (a circle, radius $=$ 100 pixels), and also to force the user to not move the mouse cursor before the target appears on the interface. The target appears randomly on the interface with a minimum distance of 400 pixels from the mouse cursor.

The user had to repeat this task 15 times. Fifteen RTs have been obtained; they are calculated in real-time for each attempt. All trajectory positions with timestamp were recorded.

\subsubsection{Dynamic Pointing}

In this procedure, and in contrast to static pointing, users can make a movement before and at the time of the target's appearance. Movement postappearance can be either directly toward the target or not. In this case, the $T_{0}$ of the equation (1) is different. In order to calculate the new $T_{0}$ and then the new RT, we created a tolerance sector using the direction vector (DVMT) between the mouse position and the target position, where each side of this tolerance sector was built with a predetermined angle $\alpha / 2$ between it and the DVMT. This tolerance sector allows us to compare the DVMT with the direction vector (DVW) of a sliding window throughout the user's trajectory. If the angle between the two vectors DVMT and DVW is less than $\alpha / 2$, we consider that users move their mouse cursor towards the target (stat B on fig. (5)) and if not, we consider that users' movement is not towards the target (stat A on fig. (5)). This method allows us to determine the beginning of the voluntary movement $T_{0}$ towards the target at any time during the execution of this dynamic pointing task.

Consequently, the RT will be described also by the equation (1) where users should move the mouse cursor until the target appears, and then the movement post-appearance should be directly toward the target. $T_{0}$ is the moment of beginning the mouse movement towards the target.

In this task, users must move the mouse cursor on the pointing application 


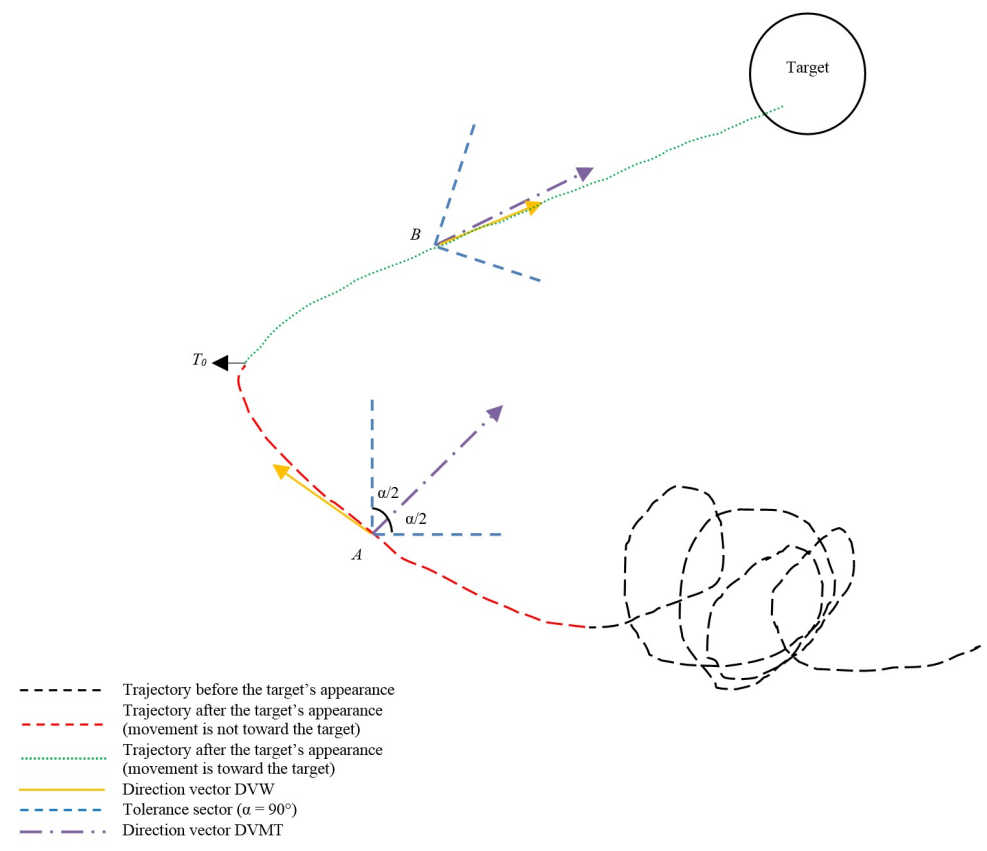

Figure 5: The mechanism of dynamic method.

interface $(1000 \times 1000$ pixels $)$ during a random period of time ranging from 1 to 3 seconds. After the target's appearance on the interface, users must make a direct movement towards the target to point at it.

The waiting period is built in order to display the target (a circle, radius $=100$ pixels), and also to force the user to move the mouse cursor before the target's appearance on the interface. The target appears randomly on the screen with a minimum distance of 400 pixels form the mouse cursor.

Users were asked to repeat this task 15 times. Fifteen RTs were obtained; they were calculated in real-time for each attempt. All trajectory positions with timestamps were recorded.

\section{Evaluation experiment of dynamic pointing}

To check the reliability of the tolerance sector that we used to calculate the moment of beginning the movement toward the target in this dynamic procedure, 
we conducted another protocol in which we asked the same subjects to move the mouse cursor during a random period of time which ranged from 1 to 3 seconds, in order to display a target (a circle, radius $=100$ pixels). Following the random appearance of the target on the interface, users must completely release the mouse. The RT here is the time interval between the target appearance and the release of the mouse. Users must repeat this procedure 15 times. We obtained 15 RTs in real time for each attempt.

\subsubsection{Game Pointing}

In this procedure (Fig. 6) we use our dynamic method to compute RT in the context of video games in order to detect user behavior during a game session.

Users played a simplified version of Le Village aux Oiseaux, where they had to find and point at all targets in this environment. Users use only a mouse to execute the required task. Players are not required to move around in the game world; the virtual camera moves automatically in the game world following a predefined path. The virtual camera pauses 20 times during the session. At each camera pause, a target (bird, radius $=100$ pixels) appears randomly on the game interface after a period of time ranging from 1 to 3 seconds. Following the target's appearance on the game interface, users must make a direct movement towards the target to point at it using the mouse.

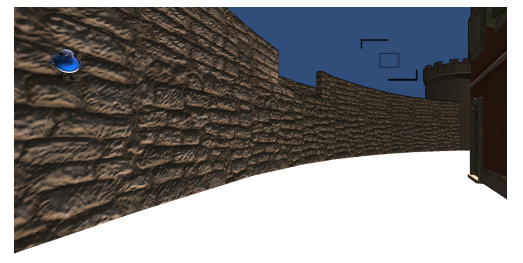

Figure 6: Game experiment: the application's interface after the target's appearance (bird).

\subsection{Results}

\subsubsection{Static Pointing Results}

We applied the equation (1) to calculate the reaction time (RT) where the user did not make any movement before the target's appearance and the move- 
ment post-appearance had to be directly toward the target. The results of this static experiment show that the RT mean of all subjects is $342 \mathrm{~ms}$ with a standard deviation of $25 \mathrm{~ms}$.

\subsubsection{Dynamic Pointing Results}

We reapplied the equation (1) to calculate the RT where the user must move the mouse cursor until the target appears, and then the movement postappearance should be directly toward the target. The figure (7) shows the RT mean for all subjects in the dynamic experiment. We used four angles of the tolerance sector $\left(\alpha=45^{\circ}, 60^{\circ}, 90^{\circ}\right.$, and $\left.120^{\circ}\right)$, so we calculated four RTs for each subject in each attempt. We can observe that the RT decreases when the tolerance sector increases.

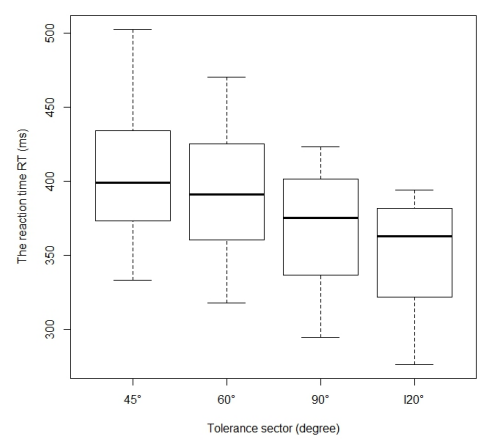

Figure 7: Dynamic experiment, mean of reaction time for all subjects

We also calculated the mean and the standard deviation of the RT for all the subjects who participated in the dynamic experiment. Table (1) shows the result of this calculation, which will be compared with the result of the evaluation experiment.

To check the reliability of the tolerance sector that we use to calculate the moment of beginning the movement toward the target in the dynamic experiment, we calculated the mean and the standard deviation of the RT obtained in the evaluation experiment (the user moves the cursor mouse to display the target and must completely release the mouse following the target's appearance). 


\begin{tabular}{|c|c|c|c|c|}
\hline Tolerance Sector & $45^{\circ}$ & $60^{\circ}$ & $90^{\circ}$ & $120^{\circ}$ \\
\hline Mean & 402 & 391 & 398 & 348 \\
Standard Deviation & 44 & 43 & 41 & 40 \\
\hline
\end{tabular}

Table 1: Dynamic experiment: mean, standard deviation of RT in ms, for all subjects

We examined whether our results were normal or not by using the Shapiro-Wilk test [36. We found that all the results we have obtained follow a normal distribution. We observed that the mean RT obtained in this evaluation experiment is 355 , with a confidence interval that varies between $298 \mathrm{~ms}$ and $413 \mathrm{~ms}$. If we look back at the table (1), we note that for a tolerance sector angle of $45^{\circ}, 60^{\circ}$, $90^{\circ}$, and $120^{\circ}$ we obtained correct values for the RT.

To determine the minimum angle of the tolerance sector that detects the moment of beginning the movement toward a target during a pointing task, we conducted an off-line analysis; by this we mean we re-analyzed the data obtained in the dynamic experiment for each subject to determine their RT but this time using a tolerance sector with an angle less than $45^{\circ}$. We observed that when we used a tolerance sector of $40^{\circ}$ we obtained correct values for the $\mathrm{RT}$, because the mean of RT obtained in this off-line analysis is $409 \mathrm{~ms}$ and it belongs to the confidence interval. When we used a tolerance sector of $35^{\circ}$, however, we observed that we obtained incorrect values for the RT because the mean of RT obtained in this off-line analysis is $419 \mathrm{~ms}$ and it does not belong to the confidence interval.

The figure (8) shows the RT mean obtained from the Dynamic experiment $\left(45^{\circ}, 60^{\circ}, 90^{\circ}\right.$, and $\left.120^{\circ}\right)$, the RT mean obtained from the off-line analysis (tolerance sector is $40^{\circ}$ ) and the confidence interval of RT obtained during the evaluation experiment.

We analyzed the angle (Ang) between the direction vector of the mouse and the direction vector between the mouse position and the target position. This analysis is intended to describe the RT values, knowing that the target response is launched in the brain before the user corrects his or her trajectory for pointing 


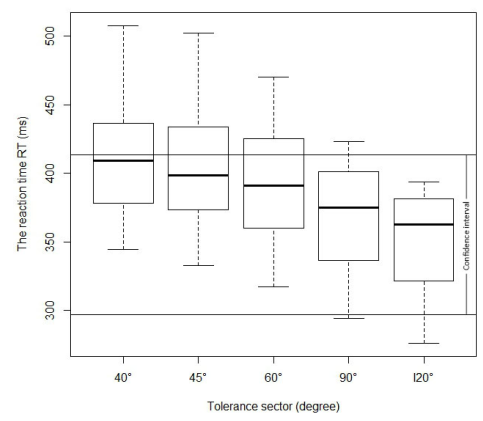

Figure 8: The RT mean obtained in the dynamic experiment with the confidence interval from the evaluation experiment

at the target. The Ang provides a way to know why the RT of a user takes, for example, $300 \mathrm{~ms}$ in the first attempt and $500 \mathrm{~ms}$ in the second attempt: it is because the Ang in the second attempt was wider than the Ang in the first. We note that the Ang mean of all users is $94^{\circ}$.

\subsubsection{Game Pointing Results}

In this last experiment, we obtained 20 RTs for each subject based on the equation (1) (using a tolerance sector angle of $90^{\circ}$ ). We considered the 5 first attempts as belonging to a calibration phase. We then calculated the mean of the 5 attempts of the calibration phase. The 15 remainder RTs were compared to the mean of the 5 attempts obtained in the calibration phase. If a RT among the remaining 15 passes above the mean of calibration, we considered that the user had a lack of attention. If a RT among the remaining 15 was lower than the mean of calibration, we considered that the user reacted normally when faced with the targets.

We did not observe a strong lack of attention in our game experiment because our subjects were healthy (non Alzheimer's patients) and could perform the experiment (with 20 attempts) with no attention-related issues. 


\subsection{Discussion}

As discovered in earlier studies, sensor-based systems have been used to measure visual attention for many years. These systems have provided many visual attention measurements useful for analyzing users' behavior. They have also shown themselves to offer a very good way of evaluating the design of interactive applications, e.g. video games.

However, the use of sensors can be intrusive, e.g. users of video games prefer a comfortable installation when they are playing video games, making the use of sensors like eye trackers, EEG, or ECG sensor unsuitable for users of video games.

The use of an indirect method for monitoring users of video games, for example, can be an interesting non-intrusive approach for the study of user behavior towards improving the design of interactive applications - such as, for example, improving navigation in virtual environments or playing videos games.

Our method uses a novel, non-intrusive technique for studying user behavior in a serious game, by analyzing the trajectory made by users using the mouse as a pointing device. This technique has allowed us to measure and evaluate the reaction time produced by users when they try to achieve pointing tasks.

Our results show that the mean of the RT mean values in the static experiment is $342 \mathrm{~ms}$ with a confidence interval that ranges between 292 and $392 \mathrm{~ms}$. If we compare the RT values obtained in the dynamic experiment with those obtained in the static experiment, we can refer back to the table (1) and find that we have RT values in the dynamic experiment (the mean of the RT mean is 390 for $60^{\circ}, 368$ for $90^{\circ}$ and 348 for $120^{\circ}$ as tolerance sector) that belong to the confidence interval of the static experiment. However, we cannot compare the static procedure with the dynamic procedure because the experiment protocols are different; this difference is expected, due to the difference in cognitive process between these two experiments.

In the case of the dynamic experiment (free pointing), we observe via figure (7) that the RTs do not have the same values for all tolerance sector angles. We deduce that the RT decreases as the tolerance sector angle increases. We notice 
that the mean of the RT values obtained in the evaluation experiment is 355 with a confidence interval that varies between $298 \mathrm{~ms}$ and $413 \mathrm{~ms}$, where we can note through the figure (8) that we can compute correct values of RT using a tolerance sector angle with a minimum value of $40^{\circ}$ in a complex environment where users can perform their trajectories without any geometric condition.

Consequently, we notice that we can compute the RT during pointing movement using only a mouse to complete a complex pointing task. RT measurement can give us significant results in terms of evaluating user attention. If the RT measure is small, we can deduce that the user reacts quickly when faced with a given task; if the RT measure is large, we can deduce that user reacts slowly when faced with this task.

We did not observe any significant amount of lack of attention (using RT as an indicator of attention) during our game experiment because our subjects were in good health and could therefore perform the task free of any attention-related problems, where the number of attempts was rather limited (20 attempts per subject). We note that this phase of the experiment was very short and for that reason it did not show a significant results; it should be extended to a longer phase that will allow us to effectively monitor user attention, especially that of Alzheimer's patients.

We have conducted experiments using our method with a unique, nonmoving target. As the results we obtained are promising, we now plan to evaluate our method with the complete version of Le Village aux Oiseaux. In the full version of the game, numerous birds can appear simultaneously, they can fly or walk, and even disappear before the player has had a chance to take a picture of them. Additionally, the final version of the game will be played with a WiiMote-like controller for accessibility and usability reasons. Thus, we will need to test our method under such conditions in order to evaluate whether it can measure RT in a similarly less-controlled environment. 


\section{Conclusion And Future Work}

In this paper, we have presented our experiments in computing reaction time as an attention factor, using a real-time, non-invasive measurement method. We applied our method to a serious game called Le Village aux Oiseaux, where pointing movements are made freely within the space.

We used reaction time to monitor user attention during the experiments, given that the reaction time analysis is important for the detection of cognitive decline and the improvement of processing speed.

We demonstrated a static method that computes reaction time that corresponds to the time interval between the target's appearance and the beginning of the movement towards the target, where the user does not make any movement before the target's appearance.

Additionally, we showed a dynamic method which computes the reaction time in a complex environment with a tolerance sector angle of $40^{\circ}$. The participants in our experiment were able to better perform a pointing task when the tolerance sector of the pointing device was larger. We showed that we can provide an attention indicator and capture the phase in which the player has begun to lose his or her attention during our game experiment. We did not observe a strong lack of attention during our video game experiment due to the fact that our subjects were healthy and the number of attempts was quite limited.

The visual attention method used in our experiment is adequate for deriving data that can be applied towards the testing of future serious games. Analyzing reaction time can be of benefit to game designers, allowing them to improve gameplay and manage the difficulty of game environments.

For future work, we plan to test our application with Alzheimer's disease patients to show how our model can actually be used in the service of cognitive rehabilitation; specifically, for facilitating pointing tasks and adapting the difficulty of a virtual environment. 


\section{References}

[1] B. Cadet, Psychologie cognitive, In Press Editions, 1998.

[2] S. Mader, S. Natkin, G. Levieux, How to analyse therapeutic games: The player / game / therapy model, in: Entertainment Computing, Vol. 7522 of ICEC, 2012, pp. 193-206.

[3] Mcgraw-hill concise dictionary of modern medicine, http://medicaldictionary.thefreedictionary.com/therapy (last access 04/2015) (2002).

[4] E. Guardiola, S. Natkin, D. Soriano, E. Loaser, P. Vrignaud, Du jeu utile au jeu serieux, Hemes 62 (2012) 87-93. doi:10.4267/2042/48283,

[5] J. Juul, The game, the player, the world: Looking for a heart of gameness, in: Digital Games Research Conference, Utrecht: Utrecht University, 2003, pp. 30-45, (last access 04/2015).

URL http://www.jesperjuul.net/text/gameplayerworld/

[6] A. C. S. Torres, Cognitive effects of videogames on older people, International Journal on Disability and Human Development 10 (2011) 55Ű58. doi:10.1515/ijdhd.2011.003.

[7] W. R. Boot, A. F. Kramer, D. J. Simons, M. Fabiani, G. Gratton, The effects of video game playing on attention, memory, and executive control, Acta Psychologica 129 (2008) 387-398. doi:10.1016/j .actpsy.2008.09. 005 .

[8] J. E. Clark, A. K. Lanphear, C. C. Riddick, The effects of videogame playing on the response selection processing of elderly adults, Journal of Gerontology 42 (1987) 82-85. doi:10.1093/geronj/42.1.82.

[9] R. E. Dustman, R. Y. Emmerson, L. A. Steinhaus, D. E. Shearer, T. J. Dustman, The effects of videogame playing on neuropsychological performance of elderly individuals., Journal of Gerontology 47 (1992) 168-171. doi:10.1093/geronj/47.3.P168. 
[10] H. Jimison, M. Pavel, J. McKanna, J. Pavel, Unobtrusive monitoring of computer interactions to detect cognitive status in elders, IEEE Transactions on Information Technology in Biomedicine 8 (3) (2004) 248-252. doi:10.1109/TITB.2004.835539.

[11] J. W. Burke, M. D. J. McNeill, D. K. Charles, P. J. Morrow, J. H. Crosbie, S. M. McDonough, Optimising engagement for stroke rehabilitation using serious games, Vis. Comput. 25 (2009) 1085-1099. doi: $10.1007 / \mathrm{s} 00371-009-0387-4$

[12] P. M. Kato, S. W. Cole, A. S. Bradlyn, B. H. Pollock, A video game improves behavioral outcomes in adolescents and young adults with cancer: A randomized trial, Pediatrics 122 (2) (2008) e305e317. arXiv:http://pediatrics.aappublications.org/content/122/ 2/e305.full.pdf+html, doi:10.1542/peds.2007-3134.

[13] A. Lenhart, S. Jones, A. R. Macgill, Adults and video games. pew internet and american life project., http://www.pewinternet.org (last access 04/2015) (December 2008).

[14] E. S. Association, Essantial facts about the computer and video game industry, (last access 04/2015) (2013).

URL http://www . theesa.com/facts/pdfs/esa_ef_2013.pdf

[15] N. Lazzaro, Why we play games: Four keys to more emotion without story, in: Game Developers Conference, 2004, pp. 1-8.

[16] N. Yee, Motivations for play in online games, CyberPsychology \& behavior 9 (6) (2006) $772-775$.

[17] E. Adams, Fundamentals of Game Design, second edition, New Riders Publishing, 2009.

[18] M.-V. Aponte, G. Levieux, S. Natkin, Difficulty in videogames: an experimental validation of a formal definition, in: Proceedings of the 8th 
International Conference on Advances in Computer Entertainment Technology, ACE 11, ACM, New York, NY, USA, 2011, pp. 49:1-49:8. doi: $10.1145 / 2071423.2071484$.

[19] C. S. Green, D. Bavelier, Action video game modifies visual selective attention, Nature PublishingGroup 423 (2003) 534-537.

[20] M. Dye, C. Green, D. Bavelier, The development of attention skills in action video game players, Neuropsychologia 47 (8-9) (2009) 1780-1789.

[21] W. Ijsselsteijn, H. Nap, Y. de Kort, K. Poels, Digital game design for elderly users, in: Proceedings of the 2007 conference on Future Play, no. 17-22 in Future Play 07, ACM, New York, NY, USA, 2007.

[22] D. Johnson, J. Wiles, P. Sweester, K. Hollingsworth, J. Gardner, The inherent appeal of physically controlled peripherals, in: International Workshop on Entertainment Computing, Makuhari, Japan, 2002, pp. 371-378.

[23] M. Csikszentmihalyi, Flow: The Psychology of Optimal Experience Harper Perennial, 1991.

URL http://www.amazon.ca/exec/obidos/redirect?tag= citeulike04-20\{\&\}path=ASIN/0060920432

[24] L. C. V. d. Andrade, L. A. V. Carvalho, C. Lima, A. Cruz, P. Mattos, C. Franco, A. Soares, B. Grieco, Supermarket game: An adaptive intelligent computer game for attention deficit/hyperactivity disorder diagnosis, in: Proceedings of the Fifth Mexican International Conference on Artificial Intelligence, Washington, DC, USA, 2006, pp. 359-368. doi:10.1109/ MICAI.2006.45,

[25] B. Seltzer, P. Zolnouni, M. Nunez, R. Goldman, D. Kumar, J. Ieni, S. Richardson, Efficacy of donepezil in early-stage alzheimer disease : a randomized placebo controlled trial, Arch Neurol 61 (12) (2004) 1852-1856. doi:10.1001/archneur.61.12.1852 
[26] E. Braverman, Cognitive decline of aging: Important neuroendocrinological predictors of early cognitive decline in a clinical setting, Weill Cornell Medical Center, PATH Medical (2011) 37-46(last access 04/2015).

URL https://www .worldhealth.net/pdf/Braverman_Thera11.pdf

[27] P. Viviani, C. Terzuolo, Trajectory determines movement dynamics, Neuroscience 7 (2) (1982) 431-437. doi:10.1016/0306-4522(82)90277-9.

[28] J. P. Hourcade, K. B. Perry, A. Sharma, Pointassist: helping four year olds point with ease, in: Proceedings of the 7th international conference on Interaction design and children, IDC 08, ACM, New York, NY, USA, 2008, pp. 202-209. doi:10.1145/1463689.1463757.

[29] P. I. Khalid, J. Yunus, R. Adnan, Extraction of dynamic features from hand drawn data for the identification of children with handwriting difficulty, Research in Developmental Disabilities 31 (2010) 256-262. doi:10.1016/ j.ridd.2009.09.009.

[30] N. Renau-Ferrer, C. Remi, Procedural analysis of a sketching activity: principles and applications, in: Proc. of the International Conference on Frontiers in Handwriting Recognition, 2012, pp. 461-466. doi:10.1109/ICFHR. 2012.255 .

[31] J. Wonner, J. Grosjean, A. Capobianco, D. Bechman, Speed : Prediction de cibles, in: Proc. of the 23rd French Speaking Conference on Human Computer Interaction, IHM 11, 2011. doi:10.1145/2044354.2044378.

[32] P. M. Fitts, The information capacity of the human motor system in controlling the amplitude of movement, Journal of Experimental Psychology 47 (6) (1954) 381-391. doi:10.1037/h0055392.

[33] L. Gueye, E. Legallet, F. Viallet, E. Trouche, G. Farnarier, Spatial orienting of attention: a study of reaction time during pointing movement, Neurophysiologie clinique 32 (6) (2002) 361-368. 
[34] M. Leclercq, J. P. Peters, BAWL Batterie Attention William Lennox, Centre Neurologique William Lennox, B.1340 Ottignies Louvain la Neuve, 2007.

URL https://www.unamur.be/medecine/psychologie/recherche/ projets-detailles/projet-bawl/presentation-bawl

[35] P. Zimmermann, B. Fimm, Tests of attentional performance (tap), http://www.psytest.net/index.php (last access 04/2015), version 2.3 (1994).

[36] S. S. Shapiro, M. B. Wilk, An analysis of variance test for normality (complete samples), Biometrika 52 (1965) 591-611.

URL http://links . jstor . org/sici?sici=0006-3444\%28196512\%2952\% 3A3\%2F4\%3C591\%3AAAOVTF $\% 3$ E2.0.CO\%3B2-B 